\title{
Laboratory diagnostic value of neopterin measurements in patients with COVID-19 infection
}

https://doi.org/10.1515/pteridines-2021-0001

received December 4, 2020; accepted December 23, 2020.

\begin{abstract}
The new coronavirus SARS-CoV-2 was identified to be responsible for the COVID-19 pandemic. There are striking differences in the response to infection, some people develop no or mild symptoms, while other outcomes are severe of even fatal. For those COVID-19 patients who require hospitalization, prognostic markers could help clinicians to identify patients with a poor outcome early. The serum levels of the immune activation marker neopterin have already been shown to be of prognostic value in patients with SARS-CoV-1 and a similar pattern can be observed for SARS-CoV-2. This comment discusses the biochemical circuits that support the clinical value of neopterin measurements in COVID-19 patients.
\end{abstract}

Keywords: COVID-19; Neopterin; Laboratory diagnostic.

\section{Introduction}

At the end of 2019 the coronavirus SARS-CoV-2 was identified to be responsible for the new COVID-19 pandemic [1]. From its starting point in south-east China it was rapidly spreading worldwide. Now at the begin of November 2020, there have been more than 45 million confirmed cases of COVID-19, 1.2 million of deaths were reported to WHO [2]. There are striking differences in the response to infection, some people develop no or mild symptoms, while other outcomes are severe of even fatal.

There is limited information about prognostic markers that could help clinicians to identify COVID-19 patients that are likely to develop a poor outcome. The serum levels of the immune activation marker neopterin have shown to be of prognostic value in patients with SARS-CoV-1 [3] and first studies provide hints that serum neopterin could be helpful in stratifying SARS-CoV-2 patients [4-6]. The aim of this commentary was to investigate whether neopterin measurements are of clinical value in COVID-19 patients.

\section{Neopterin}

Neopterin, the pyrazino-pyrimidine compound 6-D-erythro-trihydroxypropyl pterin (Fig.1) derives from guanosine triphosphate (GTP) when enzyme GTP-cyclohydrolase-1 (GCH-1) in a first step produces 7,8-dihdroneopterin triphosphate and this intermediate product is oxidized and dephosphorylates to neopterin [7]. In various human cells and cells from other species GCH-1 is stimulated by pro-inflammatory cytokines preferentially upon stimulation with cytokine interferon- $\gamma$, which is characteristic for Th1-type immune response. Thereby neopterin and its less stable sister compound 7,8-dihdroneopterin $\left(\mathrm{NH}_{2}\right)$ are produced in parallel. Depending on the environmental condition the compounds can act as a pro- or antioxidant, and both do interfere with oxidizing chemicals such as hydrogen peroxide, neopterin enhancing the oxidizing capacity whereas $\mathrm{NH}_{2}$ usually does the opposite [8].

Increased amounts of neopterin are produced by human monocyte-derived cells like macrophages and dendritic cells upon stimulation with pro-inflammatory stimuli like lipopolysaccharide and cytokines, most potently by interferon-(IFN)- $\gamma$ [9]. Other cells and cells<smiles>Nc1nc2ncc(C(O)C(O)CO)nc2c(=O)[nH]1</smiles>

Figure 1: Neopterin (D-erythro-1‘2‘3‘-trihydroxypropylpterin).
*Corresponding author: Dietmar Fuchs, Institute of Biological Chemistry, Medical University of Innsbruck, Innsbruck, Austria, E-mail: dietmar.fuchs@i-med.ac.at

Magnus Gisslen, Department of Infectious Diseases, Institute of Biomedicine, Sahlgrenska Academy, University of Gothenburg, Gothenburg, Sweden
This work is licensed under the Creative Commons Attribution alone 4.0 License. 
of other species are less able of doing so. In patients, increased neopterin concentrations were documented in body fluids like serum, urine and cerebrospinal fluid (CSF), e.g., during cytokine therapies but also in diseases that are associated with activation of the Th1-type immune system such as mycobacterial infections, autoimmune pathologies and various forms of cancer and with viral infections including HIV-1 and SARS-CoV-1 $[3,10,11]$ and recently also with SARS-CoV-2 [4-6].

In general, higher serum neopterin concentrations have been found to be associated with disease progression and reduced survival expectation and thus predict worse prognosis. In virus infections like HIV-1 but also in, e.g., cytomegalovirus (CMV) infection, neopterin levels raise during the acute phase of infection very early and decline as early as specific antibody production becomes detectable. Usually neopterin levels become normal, but HIV-1 infection is different because the vast majority of individuals remains with neopterin levels outside normal range of healthy controls. Moreover, neopterin concentrations usually correlate with virus load.

Similar to HIV-1 infection, elevated neopterin concentrations in serum and CSF are common also in patients with SARS-CoV-2 infection, and are predictive for the course of the infection [4-6]. However, in SARSCoV-2 infection, an unusual pattern of CSF inflammation is commonly present with increased soluble markers like neopterin and beta2-microglobulin while white cell response and other immunologic features typical of CNS viral infections are absent [5].

In cell culture experiments oxidizing effects of neopterin were found to enhance the production of other pro-inflammatory cytokines. Moreover, the chemical effects of ROS were further enhanced in the presence of neopterin [8]. Neopterin might represent another prooxidant in the mixture of redox-active compounds in the microenvironment of activated monocytic cells and play a role in the development of the so-called cytokine storm. The pro-inflammatory response is involved in accelerating cell metabolism and thereby most probably also virus replication.

In this study [6], neopterin concentrations were measured in sera obtained from patients with acute COVID19 infection applying a commercially available ELISA kit (BRAHMS, Hennigsdorf, Germany). The detection limit is $2 \mathrm{nmol} / \mathrm{L}$ and the upper limit of the normal is set at $10 \mathrm{nmol} / \mathrm{L}$.

At the begin all patients with acute COVID-19 infection presented with elevated neopterin levels. More than twofold higher average neopterin concentrations were found in severely ill patients compared to patients with mild symptoms, and the levels gradually decreased during the course of COVID-19, but severe cases maintained elevated levels for a longer period [6]. Serum neopterin concentrations correlated with IFN- $\gamma$ levels $(r=0.8, p$ $<0.001 ;[6])$.

\section{Tryptophan breakdown}

When stimulated monocyte-derived cells produce neopterin upon stimulation with IFN- $\gamma$ also enzyme indoleamine 2,3-dioxygenase (IDO-1) is activated at the same time. Accordingly, patients with elevated neopterin concentrations present with an increased kynurenine/ tryptophan ratio (Kyn/Trp), an index of tryptophan breakdown [12,13]. The measurement of tryptophan breakdown is done by chromatographic methods like HPLC with monitoring of tryptophan and kynurenine concentrations in parallel [12].

The activation of IDO-1 during immune response is directed against cell proliferation as a host defense strategy. Thereby unwanted proliferation of, e.g., malignant cells is suppressed. However, there are side effects caused by tryptophan starvation such as parallel inhibition of T-cell proliferation and diminished production of tryptophan metabolites like neurotransmitter 5-hydroxytryptamin (serotonin). In addition, several compounds of the so-called kynurenine pathway are likely to accumulate, among them the neurotoxin quinolinic acid.

Increased Kyn/Trp levels are common in SARSCoV-2 infection and correlate closely with enhanced neopterin concentrations, and like neopterin also Kyn/ Trp is associated with unfavorable outcomes [6]. Similar observations have been made in patients with HIV-1 infection $[13,14]$.

\section{Biochemistry related to increased neopterin production}

Cytokine IFN- $\gamma$ induces high level output of reactive oxygen species, (ROS) in cells [15]. This represents another aspect of the anti-proliferative activity of this particular cytokine, the denomination of interferons like IFN- $\gamma$ reflecting their property to induce pathways that interfere with cell proliferation. Overwhelming ROS production will wipe out the pool of antioxidants including vitamins and deficiencies of antioxidant vitamins, e.g. vitamin-E, -B and -D may develop [16, 17]. As a consequence of a developing B-vitamin deficiency, increased amounts of 
homocysteine are common in patients. Supplementation of deficient vitamins may become necessary for normal cellular functioning. Thereby, homocysteine levels often correlate with neopterin concentrations [17,18], but unlike homocysteine, neopterin concentrations are much less influenced by nutrition and are more closely associated with immune activation. Pro-inflammatory effects of neopterin have also been observed in stimulated polymorphnuclear cells (PMNs) [19]. IFN- $\gamma$ is a potent stimulus for ROS production also in PMNs, but these cells do hardly release neopterin.

The availability of data and literature about neopterin as a suitable biomarker in virus infections is very broad, especially its application in HIV infection. In Austria, even the additional screening of blood donations is mandatory since 1993 to increase infection safety.

In a similar way, iron metabolism begins to suffer from the starvation strategy of the immune system [20]. The increased amounts of pro-inflammatory cytokines induce several biochemical steps which initiate the shift of iron from the circulation into storage sites such as ferritin. This scenario is reflected by significant associations of elevated neopterin levels with declining transferrin and increasing ferritin concentrations in patients.

The overwhelming production of ROS during the immune response can trigger redox-sensitive biochemical pathways including the formation of proinflammatory cytokines, which further accelerate this vicious cycle and may end in a cytokine storm situation. Immunosuppressants like corticosteroids or antiinflammatory drugs may be the only choice to compete, but the timepoint of intervention may be critical, too.

\section{Laboratory diagnostic application of neopterin in SARS-Cov-2 infection}

Data indicates that therapeutic responses are reflected by corresponding changes of neopterin concentrations. This preliminary observation corresponds well to earlier findings in patients with SARS-1 infections or also Dengue virus infections [21], in whom therapeutic efficacy was associated with declining neopterin concentrations. Moreover, in SARS-1 infection neopterin concentrations predict duration of fever [3].

The additional measurement of neopterin concentrations is able to provide useful information in patients with prevalent SARS-CoV-2 infection. Elevated neopterin concentrations appear to relate to a productive infection [6] and thus to an increased infectious situation, whereas a low or normal neopterin is indicative for silent infection without or with less active virus production. However, further studies are still needed to be performed to confirm this conclusion which is still preliminary.

Conflict of interest: Authors state no conflict of interest

Data Availability Statement: Data sharing is not applicable to this article as no datasets were generated or analysed during the current study.

\section{References}

1. Wu Z, McGoogan JM. Characteristics of and important lessons from the coronavirus disease 2019 (COVID-19) outbreak in China: Summary of a report of 72314 cases from the Chinese Center for Disease Control and Prevention. JAMA. 2020 Apr;323(13):1239-42.

2. WHO coronavirus disease (COVID-19) dashboard. 2020, https:// covid19.who.int

3. Zheng B, Cao KY, Chan CP, Choi JW, Leung W, Leung M, et al. Serum neopterin for early assessment of severity of severe acute respiratory syndrome. Clin Immunol. 2005 Jul;116(1):1826.

4. Ozger HS, Dizbay M, Corbacioglu SK, Aysert P, Demirbas Z, Tunccan OG, et al. The prognostic role of neopterin in COVID-19 patients. J Med Virol. 2020 Aug;jmv.26472.

5. Edén A, Kanberg N, Gostner J, Fuchs D, Hagberg L, Andersson LM, et al. CSF biomarkers in patients with COVID-19 and neurological symptoms: A case series. Neurology. 2020 Oct;10:1212.

6. Robertson J, Gostner JM, Nilsson S, Andersson LM, Fuchs D, Gisslen M. Serum neopterin levels in relation to mild and severe COVID-19. BMC Infect Dis. 2020 Dec;20(1):942.

7. Werner ER, Blau N, Thöny B. Tetrahydrobiopterin: biochemistry and pathophysiology. Biochem J. 2011 Sep;438(3):397-414.

8. Weiss G, Fuchs D, Hausen A, Reibnegger G, Werner ER, WernerFelmayer $\mathrm{G}$, et al. Neopterin modulates toxicity mediated by reactive oxygen and chloride species. FEBS Lett. 1993 Apr;321(1):89-92.

9. Huber C, Batchelor JR, Fuchs D, Hausen A, Lang A, Niederwieser $D$, et al. Immune response-associated production of neopterin. Release from macrophages primarily under control of interferon-gamma. J Exp Med. 1984 Jul;160(1):310-6.

10. Fuchs D, Hausen A, Reibnegger G, Werner ER, Dierich MP, Wachter H. Neopterin as a marker for activated cell-mediated immunity: application in HIV infection. Immunol Today. 1988 May;9(5):150-5.

11. Murr C, Widner B, Wirleitner B, Fuchs D. Neopterin as a marker for immune system activation. Curr Drug Metab. 2002 Apr;3(2):175-87.

12. Widner B, Werner ER, Schennach H, Wachter H, Fuchs D. Simultaneous measurement of serum tryptophan and kynurenine by HPLC. Clin Chem. 1997 Dec;43(12):2424-6. 
13. Schröcksnadel K, Wirleitner B, Winkler C, Fuchs D. Monitoring tryptophan metabolism in chronic immune activation. Clin Chim Acta. 2006 Feb;364(1-2):82-90.

14. Sucher R, Kurz K, Margreiter R, Fuchs D, Brandacher G. Antiviral activity of interferon-g involved in impaired immune function in infectious diseases. Pteridines. 2013;24(3):149-64.

15. Nathan CF, Murray HW, Wiebe ME, Rubin BY. Identification of interferon-gamma as the lymphokine that activates human macrophage oxidative metabolism and antimicrobial activity. J Exp Med. 1983 Sep;158(3):670-89.

16. Gostner JM, Becker K, Ueberall F, Fuchs D. The good and the bad of antioxidants. Food Chem Toxicol. 2015;80:72-9.

17. Fuchs D, Jaeger M, Widner B, Wirleitner B, Artner-Dworzak E, Leblhuber $F$. Is hyperhomocysteinemia due to the oxidative depletion of folate rather than to insufficient dietary intake? Clin Chem Lab Med. 2001 Aug;39(8):691-4.

18. Ploder M, Schroecksnadel K, Spittler A, Neurauter G, Roth E, Fuchs $D$. Moderate hyperhomocysteinemia in patients with multiple trauma and with sepsis predicts poor survival. Mol Med. 2010;16:498-504.

19. Razumovitch JA, Fuchs D, Semenkova GN, Cherenkevich SN. Influence of neopterin on generation of reactive species by myeloperoxidase in human neutrophils. Biochim Biophys Acta. 2004 Apr;1672(1):46-50.

20. Weiss G, Wachter H, Fuchs D. Linkage of cell-mediated immunity to iron metabolism. Immunol Today. 1995 Oct;16(10):495-500.

21. Chan CP, Choi JW, Cao KY, Wang M, Gao Y, Zhou DH, et al. Serum neopterin for early assessment of severity of Dengue virus infection. J Infect. 2006;53:152-8. 